\title{
Ketamine Infusion for Super Refractory Status Epilepticus in Alternating Hemiplegia of Childhood
}

\author{
Debopam Samanta ${ }^{1}$ \\ ${ }^{1}$ Child Neurology Section, Department of Pediatrics, University of \\ Arkansas for Medical Sciences, Little Rock, AR, United States \\ Neuropediatrics 2020;51:225-228.
}

\begin{abstract}
Address for correspondence Debopam Samanta, MD, Child Neurology Section, Department of Pediatrics, University of Arkansas for Medical Sciences, 1 Children's Way, Little Rock, Arkansas 72202, United States (e-mail: dsamanta@uams.edu).
\end{abstract}

\begin{abstract}
Keywords

- ketamine

- status epilepticus

- refractory

- alternating hemiplegia of childhood

Alternating hemiplegia of childhood $(\mathrm{AHC})$ is a rare neurodevelopmental disorder and characterized by infantile onset hemiplegia involving either side of the body and other paroxysmal spells, including epilepsy. The $\mathrm{N}$-methyl-D-aspartate (NMDA) receptor noncompetitive antagonist ketamine has been proved to be effective terminating status epilepticus (SE) in animal models and human case studies. Less than 50 cases, those use ketamine, have been reported in the management of pediatric SE, but its effectiveness is unknown in patients with AHC. The author reported two patients with molecularly confirmed AHC who presented with super refractory status epilepticus. These two patients failed multiple antiepileptic drugs, including midazolam infusion and propofol but responded promptly to ketamine infusion. This is the first description of the effectiveness of ketamine as an effective third-line agent for super refractory SE associated with AHC. Increased glutamate-mediated neurotoxicity can be present in AHC, not only due to overexpression of glutamate receptors during status epilepticus but also due to unique characteristics of AHC-preferential inactivity of GABAergic interneuron and impaired action of glutamate transporters (EAAT-1). Moreover, as neuroregression is possible after SE in AHC, earlier termination of SE by using ketamine can be an effective option in this population. Further prospective, controlled studies are needed to confirm the finding of this study.
\end{abstract}

Alternating hemiplegia of childhood $(\mathrm{AHC})$ is a rare neurodevelopmental disorder and characterized by infantile onset hemiplegia involving either side of the body and other paroxysmal spells, including epilepsy. Status epilepticus (SE) is reported in patients with AHC with the common occurrence of refractory SE due to failure of cessation of seizures after administration of first and second line benzodiazepine and nonbenzodiazepine antiepileptics. Use of ketamine, an emerging third-line agent for SE, in AHC patients has not been reported.

\section{Case Description}

A 6-year-old girl with molecularly confirmed AHC (E815K) admitted with episodes of picking at bed sheets and lip- smacking. She had frequent epileptic seizures and SE since early infancy. At the presentation, She was more somnolent during day time with the inability to sleep at nights and excessive irritability. She was afebrile with no evidence of recent infection. The patient had several episodes of the right arm and leg flexion, lip-smacking, followed by bilateral leg rhythmic clonic jerking after coming to the hospital. Routine laboratory, imaging, and infectious workup were unremarkable. She had several episodes of focal seizures without returning to her normal self in between and required rectal diazepam, intravenous (IV) lorazepam, fosphenytoin, levetiracetam, followed by IV midazolam and dexmedetomidine infusion for management of super refractory SE. Later, she required pentobarbital coma due to continuing electroclinical or electrographic-only seizures. She had a total 25 days

(c) 2020 Georg Thieme Verlag KG Stuttgart · New York
DOI https://doi.org/ 10.1055/s-0039-3402005. ISSN 0174-304X.

July 3, 2019 
stay in the critical care unit due to several complications associated with super refractory SE: hypotension requiring ionotropic support, intermittent bradycardia, prolonged intubation, and pleural effusion. She had prolonged wean of anesthetics with methadone, lorazepam, and clonidine. She slowly returned to her baseline except for a mild decline in her cognitive skill and worsening behavioral outbursts.

After 7 months of the previous presentation, this patient again returned with episodes of bilateral convulsion involving all four extremities in the setting of vomiting and rhinorrhea. She was on valproate, oxcarbazepine, topiramate, clonazepam, and levetiracetam, with no recent medication noncompliance. Rectal diazepam at home, followed by several doses of IV lorazepam by the fast responders did not stop these seizures. She was intubated for airway protection, and several nonbenzodiazepine IV antiseizure medications were administered that failed to control SE. Midazolam infusion $(1.5 \mathrm{mg} / \mathrm{kg} / \mathrm{h})$ controlled seizures for 36 hours with the eventual return of multiple episodes of electroclinical focal seizures. IV ketamine bolus dose $(2 \mathrm{mg} / \mathrm{kg})$ is given followed by continuous infusion $(0.5-2.5 \mathrm{mg} / \mathrm{kg} / \mathrm{h}$; -Fig. 1). The patient remained seizure free with successful weaning of anesthetic infusions over the next 48 hours and discharged from the hospital within 1 week.

A 3-year-old boy with AHC diagnosis (D801N) admitted due to recurrent episodes paroxysmal epileptic (convulsive) and nonepileptic episodes over 24 hours. He was diagnosed with AHC due to recurrent episodes of eye deviation at the age of 3 months and developed paretic episodes involving either side of the body since age 7 months. He also developed episodes of convulsive seizures since age 9 months and had multiple episodes of SE since that time. His daily home regimen of antiepileptics was a combination of levetiracetam and topiramate, and the family denied any missed doses or any other possible triggering factors except insomnia and irritability for two prior nights. At that time of presentation,
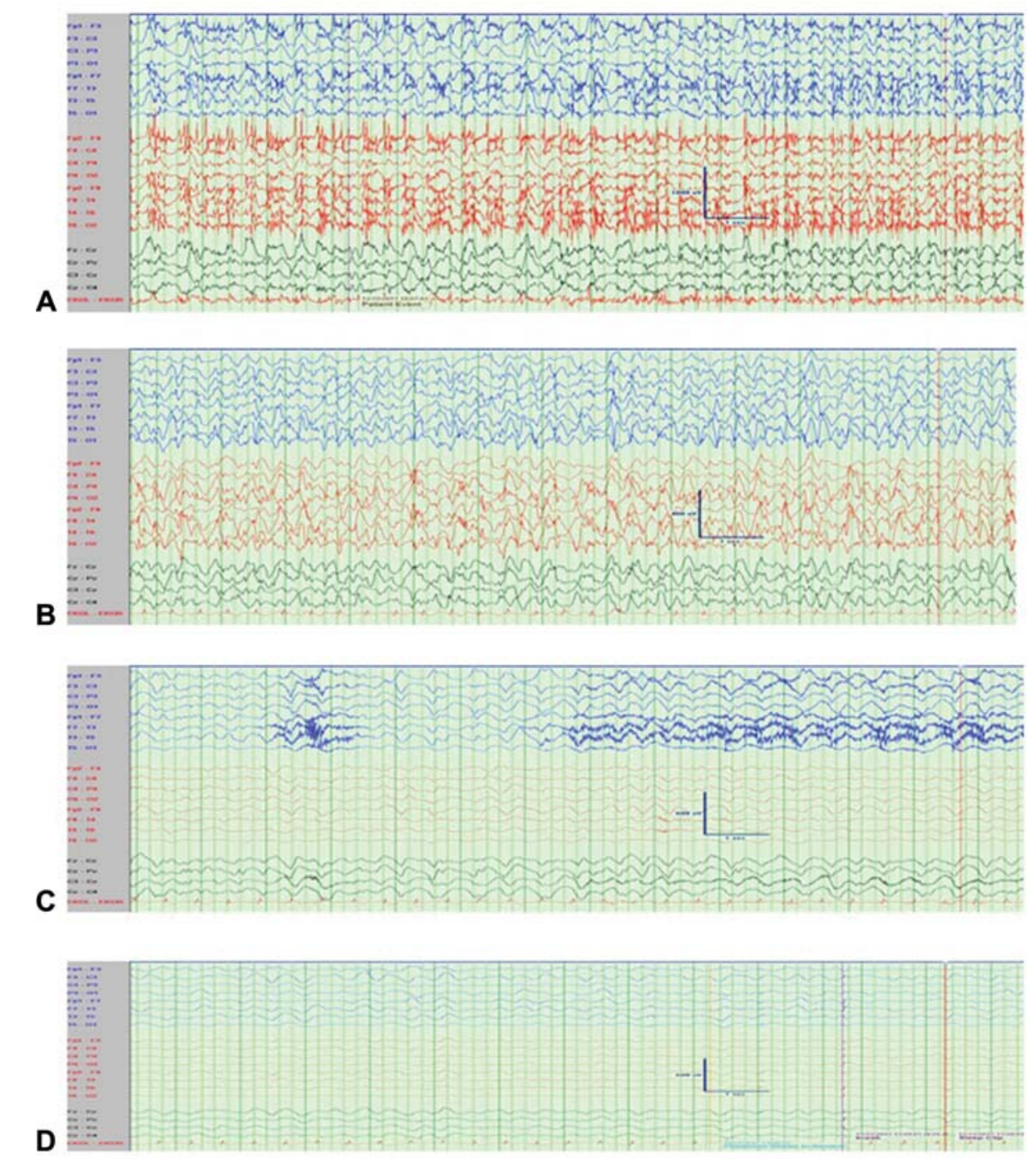

Fig. 1 (A) EEG revealed ictal rhythm of bilateral $2.5 \mathrm{~Hz}$ high amplitude sharply contoured delta activity intermixed with fast frequency discharges. EMG artifacts were due to clinical seizures. (B) Recurrent electrographic-only seizure with rhythmic high amplitude delta with admixed fast frequency discharges with a right hemispheric predominance. (C) A focal seizure started from the left hemisphere during midazolam infusion. (D) Diffuse delta slowing with mild suppression with ketamine infusion. EEG, electroencephaography; EMG, electromyography. 
he was noted to have a paucity of movement of the right side of the body with electroclinical evidence of frequent bilateral convulsive seizures (-Fig. 2). The patient was treated with intranasal midazolam and IV lorazepam, fosphenytoin, levetiracetam, lacosamide, followed by midazolam infusion $(1.2 \mathrm{mg} / \mathrm{kg} / \mathrm{h})$, and several propofol boluses due to frequent recurrent seizures. After 30 hours of recurrent seizures, ketamine bolus ( $1 \mathrm{mg} / \mathrm{kg}$ ) was given, and maintenance infusion $(0.5-2 \mathrm{mg} / \mathrm{kg} / \mathrm{h})$ was started. He had a recurrent seizure after 6 hours when additional ketamine bolus $(2 \mathrm{mg} / \mathrm{kg}$ ) was given with increment of the infusion rate to $3 \mathrm{mg} / \mathrm{kg} / \mathrm{h}$. The patient remained seizure free afterward, and no additional antiepileptic was needed to introduce before successful weaning of anesthetics after 36 hours of seizure-free period.

\section{Discussion}

Internalization of gamma aminobutyric acid (GABA) receptors from the postsynaptic surface and overexpression of glutamate receptors during SE may make GABA mediated third-line seizure agents relatively ineffective in late stages of SE. ${ }^{1}$ Additionally, abnormal network physiology has been suggested in AHC with the GABAergic interneuron activity might be preferentially affected in ATP1A3 pathogenic variants and produces an excitatory state due to glutamateGABA imbalance. ${ }^{2}$ Moreover, glutamate concentration may also increase due to impaired glutamate transporters (EAAT1), colocalized with $\mathrm{Na}^{+}-\mathrm{K}^{+}-$ATPase. Around half of the patients with AHC have epilepsy, and the majority of these patients have drug-resistant epilepsy, including both focal and generalized epilepsy. ${ }^{3}$ Approximately one-fourth of AHC patients have SE. They can have recurrent SE and refractory and super refractory SE. In a cohort of 24 patients, 6 had convulsive (focal to secondary bilateral) SE and all were refractory with one had a superrefractory event. ${ }^{4}$ These patients required more than three medications, including benzodiazepine and nonbenzodiazepine antiepileptics, to terminate the SE in a mean duration of 7.5 hours. ${ }^{4}$ Ketamine was not used in this cohort. Neurodevelopmental regression after SE episodes has been reported in AHC.

Ketamine, a N-methyl-D-aspartate (NMDA) receptor antagonist, has been proved to be effective terminating SE in animal models and human case studies. This is the first description of the effectiveness of ketamine as an effective third-line agent for super refractory SE associated with AHC. Less than 50 cases of using ketamine have been reported in the management of pediatric SE of all etiologies, and the use of both oral and IV routes was described. ${ }^{5}$ The overall success rate was estimated to be around threefourths of the pediatric patients, with $100 \%$ response noted in a case series involving patients with Lennox-Gastaut syndrome, pseudo Lennox-Gastaut syndrome, myoclonicastatic epilepsy, and progressive myoclonic epilepsy. ${ }^{6}$ In this particular case series, all children had seizure cessation within 48 hours of ketamine administration with no mortality. Rosati et al reported six of nine children with refractory status epilepticus (RSE) who responded positively to ketamine therapy. ${ }^{7}$ Side-effect profile of ketamine is

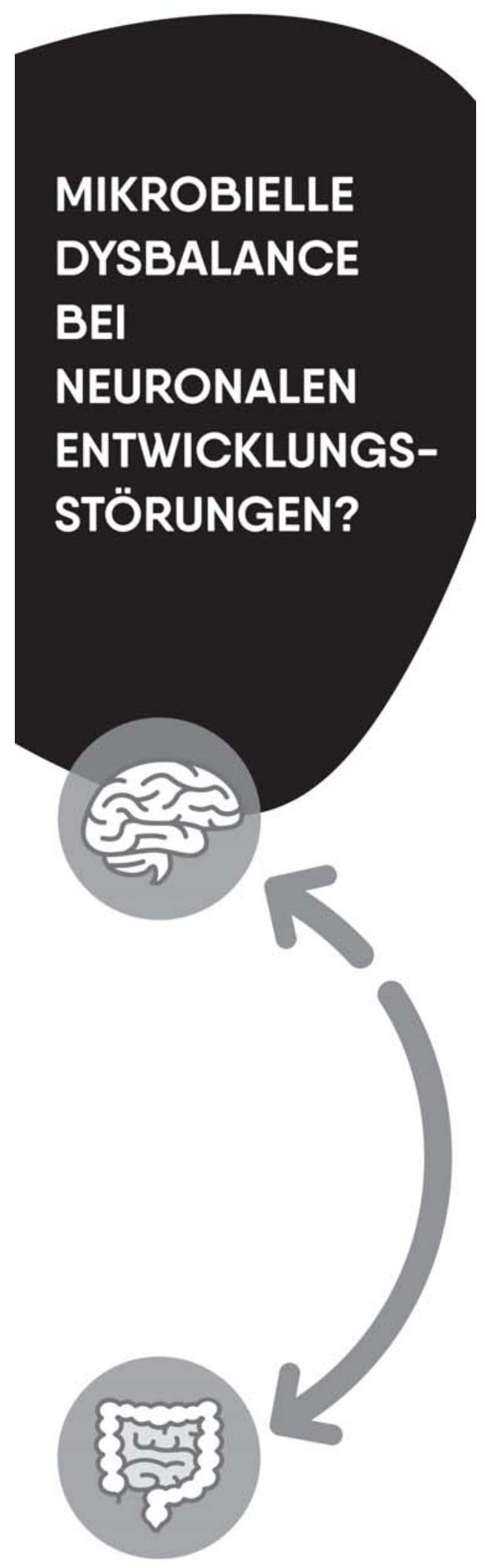

MEURAXPHARm 

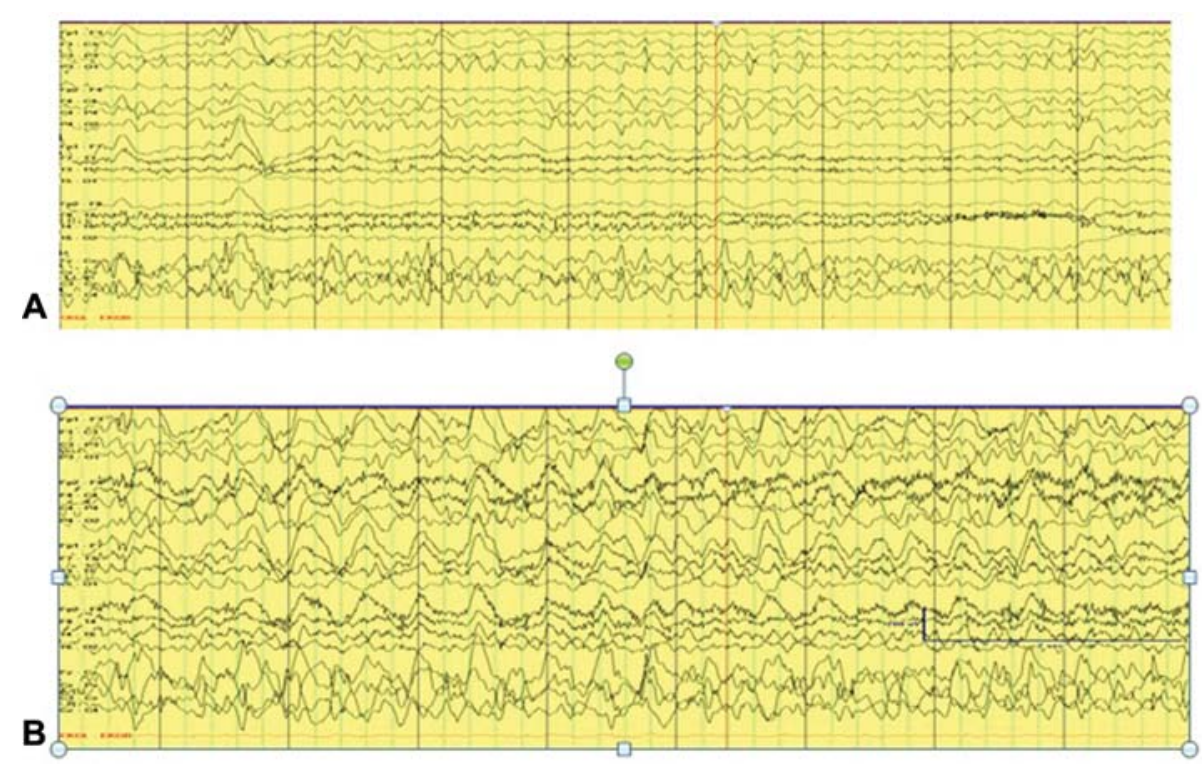

Fig. 2 (A) Fast frequency ictal rhythm was started from the midline electrodes.(B) Bilateral spread of ictal rhythm was noted with high amplitude sharply contoured delta with admixed fast frequency discharges.

attractive, and predominantly irritability and increased salivation were reported as adverse effects. Ketamine by preventing glutamate excitotoxicity may reduce $\mathrm{Ca}^{2+}$ influx and subsequent cell death. Ketamine increases blood pressure, heart rate, and cardiac output, and can counteract negative hemodynamic consequences from other anesthetics commonly used in the management of SE.

In these two patients, ketamine was used after they failed combination of IV anesthetics and continuous electroencephalography (EEG) was utilized for monitoring throughout the stay. Ketamine infusion rate was controlled to maintain seizure freedom but not to reach a definitive burst suppression pattern. Coinfusions were weaned within 24 hours of ketamine infusion and didn't require prolonged dual therapy. Ketamine was the last antiepileptic agent to be included in both the patients and no other antiepileptic agent was administered via gastrostomy tube to wean anesthetics. One of the patients required less vasopressor support after initiation of ketamine. Both patients were discharged home without requirement of inpatient rehabilitation or significant change in the cognition and behavior during shortposthospital discharge follow-up.

Though the natural resolution of seizure, irrespective of ketamine, is possible in these cases; the close temporal resolution of seizure after ketamine may indicate potential effectiveness. It is unknown if dual therapy of midazolam and ketamine had a synergistic effect in these patients. Moreover, as neuroregression is possible after SE in AHC, earlier termination of SE by using ketamine can be an effective option in this population. Although there is a growing interest in the use of ketamine infusion for SE, further prospective controlled studies are needed to determine the optimum dose, appropriate timing (early versus after the failure of conventional third-line agents), and use as third-line monotherapy versus early polytherapy with other IV anesthetics. More- over, further studies are necessary to determine if ketamine can induce neuronal cell death or alter neurogenesis. ${ }^{8}$

\section{Funding}

The author received no financial support for the research, authorship, and/or publication of this article.

\section{Declaration of Conflicting Interests}

The author declared no potential conflicts of interest concerning the research, authorship, and/or publication of this article.

\section{References}

1 Goodkin HP, Yeh JL, Kapur J. Status epilepticus increases the intracellular accumulation of GABAA receptors. J Neurosci 2005;25(23):5511-5520

2 Bøttger P, Tracz Z, Heuck A, Nissen P, Romero-Ramos M, LykkeHartmann K. Distribution of Na/K-ATPase alpha 3 isoform, a sodium-potassium P-type pump associated with rapid-onset of dystonia parkinsonism (RDP) in the adult mouse brain. J Comp Neurol 2011;519(02):376-404

3 Uchitel J, Helseth A, Prange L, et al. Epilepsy in alternating hemiplegia of childhood: characteristics, neuroimaging, and response to therapy. Neurology 2019;92(15 Suppl):P4.6-069

4 Uchitel J, Prange L, McLean M, Wuchich J, Mikati M. Characteristics of status epilepticus in alternating hemiplegia of childhood. 2017;88(16 Suppl):P3.198

5 Höfler J, Trinka E. Intravenous ketamine in status epilepticus. Epilepsia 2018;59(Suppl 2):198-206

6 Mewasingh LD, Sékhara T, Aeby A, Christiaens FJ, Dan B. Oral ketamine in paediatric non-convulsive status epilepticus. Seizure 2003;12(07):483-489

7 Rosati A, L'Erario M, Ilvento L, et al. Efficacy and safety of ketamine in refractory status epilepticus in children. Neurology 2012;79 (24):2355-2358

8 Ubogu EE, Sagar SM, Lerner AJ, Maddux BN, Suarez JI, Werz MA. Ketamine for refractory status epilepticus: a case of possible ketamine-induced neurotoxicity. Epilepsy Behav 2003;4(01):70-75 